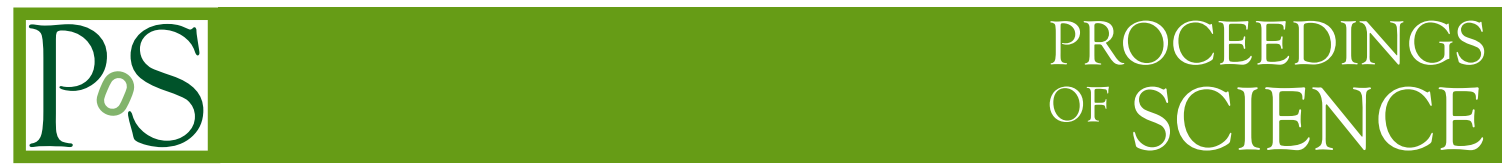

\title{
Neutrinoless double beta decay results from CUORE- 0 and status of the CUORE experiment
}

\author{
F. Terranova* \\ Università di Milano-Bicocca and INFN, Milano, Italy \\ E-mail: francesco.terranova@mib.infn.it
}

C. Alduino ${ }^{2}$, K. Alfonso ${ }^{20}$, D. R. Artusa ${ }^{1,2}$, F. T. Avignone $\mathrm{III}^{2}$, O. Azzolini ${ }^{3}$, M. Balata ${ }^{1}$, T. I. Banks ${ }^{4,5}$, G. Bari ${ }^{6}$, J. Beeman ${ }^{7}$, F. Bellini ${ }^{8,9}$, A. Bersani $^{10}$, M. Biassoni ${ }^{11,12}$, C. Brofferio $^{11,12}$, C. Bucci ${ }^{1}$, A. Camacho ${ }^{3}$, A. Caminata ${ }^{10}$, L. Canonica ${ }^{1}$, X.G. Cao ${ }^{13}$, S. Capelli $^{11,12}$, L. Cappelli ${ }^{10}$, L. Carbone ${ }^{12}$, L. Cardani ${ }^{8,9}$, P. Carniti ${ }^{11,12}$, N. Casali ${ }^{8,9}$, L. Cassina $^{11,12}$, D. Chiesa ${ }^{11,12}$, N. Chott ${ }^{2}$, M. Clemenza ${ }^{11,12}$, S. Copello ${ }^{10,15}$, C.

Cosmelli $^{8,9}$, O. Cremonesi ${ }^{12}$, R. J. Creswick ${ }^{2}$, J.S. Cushman ${ }^{14}$, I. Dafinei ${ }^{9}$, A. Dally ${ }^{14}$, C. J. Davis ${ }^{14}$, S. Dell’Oro ${ }^{1,31}$, M. M. Deninno ${ }^{6}$, S. Di Domizio ${ }^{10,15}$, M. L. di Vacri ${ }^{1,32}$, A. Drobizhev $^{4,5}$, D. Q. Fang ${ }^{13}$, M. Faverzani ${ }^{11,12}$, G. Fernandes ${ }^{10,15}$, E. Ferri ${ }^{11,12}$, F. Ferroni $^{8,9}$, E. Fiorini ${ }^{11,12}$, A. Franceschi ${ }^{34}$, S. J. Freedman ${ }^{4,5}$, B. K. Fujikawa ${ }^{5}$, A. Giachero $^{11,12}$, L. Gironi ${ }^{11,12}$, A. Giuliani ${ }^{17}$, P. Gorla ${ }^{1}$, C. Gotti ${ }^{11,12}$, T. D. Gutierrez ${ }^{18}$, E. E. Haller ${ }^{7,19}$, K. Han ${ }^{14}$, E. Hansen ${ }^{20,33}$, K. M. Heeger ${ }^{14}$, R. Hennings-Yeomans ${ }^{4,5}$, K. P. Hickerson $^{20}$, H. Z. Huang ${ }^{20}$, R. Kadel ${ }^{21}$, G. Keppel ${ }^{3}$, Yu. G. Kolomensky ${ }^{4,21}$, C. Ligi ${ }^{34}$,

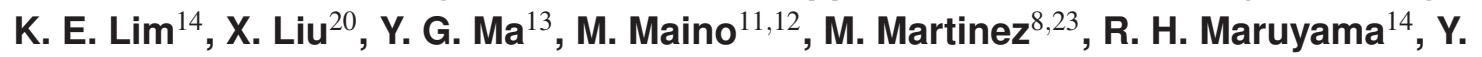
Mei $^{5}$, N. Moggi ${ }^{6,16}$, S. Morganti ${ }^{9}$, P. Mosteiro ${ }^{9}$, T. Napolitano ${ }^{34}$, S. Nisi ${ }^{1}$, C. Nones ${ }^{24}$, E. B. Norman ${ }^{22,25}$, A. Nucciotti ${ }^{11,12}$, T. O’Donnell ${ }^{4,5}$, F. Orio ${ }^{9}$, D. Orlandi ${ }^{1}$, J. L. Ouellet $^{33}$, C. E. Pagliarone ${ }^{1,34}$, M. Pallavicini ${ }^{10,15}$, V. Palmieri ${ }^{3}$, L. Pattavina ${ }^{1}$, M. Pavan $^{11,12}$, G. Pessina ${ }^{12}$, V. Pettinacci ${ }^{9}$, G. Piperno ${ }^{8,9}$, C. Pira ${ }^{3}$, S. Pirro ${ }^{1}$, S. Pozzi ${ }^{11,12}$, E. Previtali ${ }^{12}$, C. Rosenfeld ${ }^{2}$, C. Rusconi ${ }^{12}$, E. Sala ${ }^{11,12}$, S. Sangiorgio ${ }^{22}$, D. Santone ${ }^{1,32}$, N. D. Scielzo ${ }^{22}$, M. Sisti ${ }^{11,12}$, A. R. Smith ${ }^{5}$, L. Taffarello ${ }^{28}$, M. Tenconi ${ }^{17}$, C. Tomei $^{9}$, S. Trentalange ${ }^{20}$, G. Ventura ${ }^{29,30}$, M. Vignati $^{9}$, S. L. Wagaarachchi ${ }^{4,5}$, B. S. Wang $^{22,25}$, H. W. Wang ${ }^{13}$, J. Wilson ${ }^{2}$, L. A. Winslow ${ }^{33}$, T. Wise ${ }^{14}$, L. Zanotti ${ }^{11,12}$, C. Zarra $^{1}$, G. Q. Zhang ${ }^{13}$, B. X. Zhu ${ }^{20}$, S. Zimmerman ${ }^{27}$, S. Zucchelli6,26 


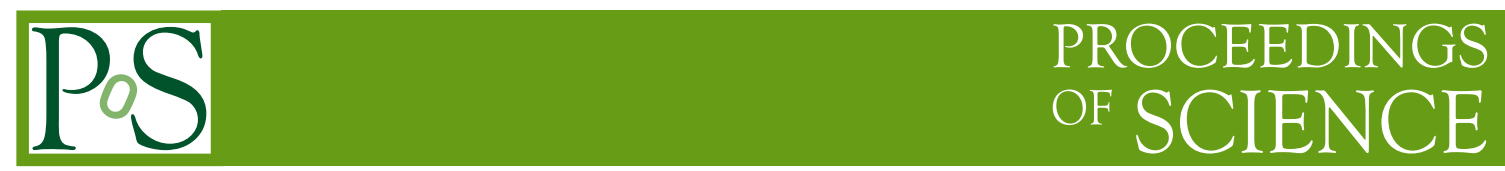

1 INFN, Laboratori Nazionali del Gran Sasso, Assergi, AQ, Italy; 2 Department of Physics and Astronomy, University of South Carolina, Columbia, SC, USA; 3 INFN - Laboratori Nazionali di Legnaro, Legnaro (Padova), Italy; 4 Department of Physics, University of California, Berkeley, CA USA; 5 Nuclear Science Division, Lawrence Berkeley National Laboratory, Berkeley, CA, USA; 6 INFN - Sezione di Bologna, Bologna, Italy; 7 Materials Science Division, Lawrence Berkeley National Laboratory, Berkeley, CA, USA; 8 Dipartimento di Fisica, Sapienza Università di Roma, Roma, Italy; 9 INFN - Sezione di Roma, Roma, Italy; 10 INFN - Sezione di Genova, Genova, Italy; 11 Dipartimento di Fisica, Università di Milano-Bicocca, Milano, Italy; 12 INFN - Sezione di Milano Bicocca, Milano, Italy; 13 Shanghai Institute of Applied Physics (Chinese Academy of Sciences), Shanghai, China; 14 Department of Physics, Yale University, New Haven, CT, USA; 15 Dipartimento di Fisica, Università di Genova, Genova, Italy; 16 Dipartimento di Scienze per la Qualità della Vita, Alma Mater Studiorum - Universitá di Bologna, Bologna, Italy; 17 Centre de Spectrometrie Nucleaire et de Spectrometrie de Masse, Orsay Campus, France; 18 Physics Department, California Polytechnic State University, San Luis Obispo, CA, USA; 19 Department of Materials Science and Engineering, University of California, Berkeley, CA, USA; 20 Department of Physics and Astronomy, University of California, Los Angeles, CA, USA; 21 Physics Division, Lawrence Berkeley National Laboratory, Berkeley, CA, USA; 22 Lawrence Livermore National Laboratory, Livermore, CA, USA; 23 Laboratorio de Fisica Nuclear y Astroparticulas, Universidad de Zaragoza, Zaragoza, Spain; 24 Service de Physique des Particules, CEA, Saclay, Gif-sur-Yvette, France; 25 Department of Nuclear Engineering, University of California, Berkeley, CA, USA; 26 Dipartimento di Fisica, Università di Bologna, Bologna, Italy; 27 Engineering Division, Lawrence Berkeley National Laboratory, Berkeley, CA, USA; 28 INFN - Sezione di Padova, Padova, Italy; 29 Dipartimento di Fisica, Università di Firenze, Firenze, Italy; 30 INFN Sezione di Firenze, Firenze, Italy; 31 INFN - Gran Sasso Science Institute, L'Aquila, Italy; 32 Dipartimento di Scienze Fisiche e Chimiche, Università dell'Aquila, L'Aquila, Italy; 33 Massachusetts Institute of Technology, Cambridge, MA, USA; 34 INFN - Laboratori Nazionali di Frascati, Frascati (Roma), Italy;

The Cryogenic Underground Observatory for Rare Events (CUORE) is a ton-scale neutrinoless double beta decay experiment based on cryogenic bolometers that is in the final stage of construction at the Gran Sasso National Laboratory (LNGS). CUORE is aimed at observing the neutrinoless double beta decay of ${ }^{130} \mathrm{Te}$ and measuring the corresponding Majorana neutrino mass with a sensitivity close to the so-called inverted mass hierarchy region. CUORE-0 is the demonstrator of CUORE: a single CUORE-like tower, made of $52 \mathrm{TeO}_{2}$ bolometers for a total mass of $39 \mathrm{~kg}$. CUORE-0 started taking data in spring 2013 at LNGS and reached a total exposure of $9.8 \mathrm{~kg}$ y of ${ }^{130} \mathrm{Te}$. CUORE- 0 significantly reduces the background and energy resolution of the previous experiment CUORICINO and sets the most stringent limit to date on neutrinoless double beta decay in ${ }^{130} \mathrm{Te}$. We will discuss in the following the physics results from CUORE- 0 data and the status of the construction of CUORE.

The European Physical Society Conference on High Energy Physics 22-29 July 2015

Vienna, Austria

\footnotetext{
*Speaker.
} 


\section{Introduction}

Neutrinoless double-beta $(0 v \beta \beta)$ decay is a lepton-number-violating process first considered by Pontecorvo in 1967 [1] and never observed so far. The observation of this process would establish the Majorana nature of neutrinos, would constrain the neutrino mass scale and hierarchy and would uniquely demonstrate the violation of the lepton number conservation. As a consequence, an intense experimental effort is ongoing to search for this decay [2,3]. The neutrinoless double-beta decay can proceed via different mechanisms. After the discovery of neutrino flavor oscillations, the preferred mechanism is the exchange of a massive Majorana neutrino.

Among the upcoming experiments searching for this rare decay, the Cryogenic Underground Observatory for Rare Events (CUORE) at the Gran Sasso National Laboratory (LNGS), is expected to be one of the most sensitive $[4,5]$. CUORE takes advantage of the bolometric technique [6] to build detectors with outstanding energy resolution. The CUORE bolometers are $5 \times 5 \times 5 \mathrm{~cm}^{3} \mathrm{TeO}_{2}$ crystals operated at a temperature of $10 \mathrm{mK}$. In a cryogenic bolometer, the energy released by an interacting particle in the crystal (absorber) is converted into lattice vibrations and induces a temperature rise $(\Delta T)$ in the device. This increase of temperature is measured by a thermometer a semiconductor thermistor - that is glued onto the surface of the absorber. The thermistor converts the $\Delta T$ into an electric pulse whose shape can be recorded and analyzed.

CUORE will search for the $0 v \beta \beta$ decay of ${ }^{130} \mathrm{Te}$, an isotope of tellurium. The expected signature is a peak in the measured energy spectrum at the transition energy $\mathrm{Q}_{\beta \beta}=2528 \mathrm{keV} . \mathrm{TeO}_{2}$ crystals have been chosen for several reasons: the excellent bolometric properties of this material, the high natural abundance of the $\beta \beta$ emitting isotope ${ }^{130} \mathrm{Te}$ (34\% - the highest among the isotopes used to date) and a quite high $\mathrm{Q}_{\beta \beta}$, nearly above the energy spectrum of natural radioactivity. In fact, $\mathrm{TeO}_{2}$ crystals have been successfully operated since more than ten years to search for the $0 v \beta \beta$ of ${ }^{130} \mathrm{Te}$. The CUORICINO experiment [7], a predecessor of CUORE, set a limit on the half-life of $0 v \beta \beta$ at $T_{1 / 2}^{0 v}>2.8 \times 10^{24} \mathrm{yr}$ at $90 \%$ C.L. CUORE will scale up the sensitivity of CUORICINO by increasing the mass, improving the energy resolution and reducing the background (see Sec. 4).

\section{CUORE-0}

CUORE-0 has been built to validate the procedures for the CUORE detector construction. It corresponds to a single CUORE-like tower and was operated in LNGS as an independent detector between March 2013 and March 2015. The CUORE-0 detector consists of 52 nat $\mathrm{TeO}_{2}$ crystals, arranged in a tower structure made of 13 floors. Each floor hosts four $5 \times 5 \times 5 \mathrm{~cm}^{3} \mathrm{TeO}_{2}, 750 \mathrm{~g}$ each. The crystals are enclosed in a copper structure, and they are held in position by means of polytetrafluoroethylene (PTFE) supports. The changes in crystal temperature are measured using the same thermistors that will be used in CUORE: a neutron-trasmutation-doped (NTD) sensor that is coupled on each individual crystal by means of glue spots. In addition, a silicon heater is also glued on each crystal. The heater is employed to monitor the stability of the detector gain, injecting a fixed amount of energy on the crystal. The whole crystal array is cooled down to a temperature of 10-12 mK, using the same dilution refrigerator located in the Hall A of the Gran Sasso National Laboratory that already hosted CUORICINO. 
CUORE-0 can be considered the demonstrator of CUORE because the crystal array was built using all the procedures that have been developed to reduce background contamination in CUORE. These procedures include raw material selection, crystal growing in controlled environment and cleaning of the detector components to mitigate surface contamination. Special care have been taken to reduce re-contamination during the detector construction and assembly: in particular, all the steps for the construction of the detector array were performed in custom made glove boxes, continuously flushed with nitrogen gas, with the aim of reducing the exposure of the clean detector components to radon.

The CUORE-0 detector was assembled in spring 2012. Data taking started in March 2013. The first set of runs were completed in September 2013, when a maintenance of the cryogenic system was performed. The data taking resumed in November 2013 with a significantly higher duty cycle and lasted until March 2015. The CUORE-0 data amounts to a total exposure of $35.2 \mathrm{~kg}$ $\mathrm{yr}$ of $\mathrm{TeO}_{2}$, corresponding to $9.8 \mathrm{~kg}$ yr of ${ }^{130} \mathrm{Te}$. The duty cycle of the detector was $78.6 \%$. Physics data taking corresponds to $64 \%$ of the whole data taking of the experiment.

The performance of CUORE- 0 were extremely good in terms of energy resolution and background reduction. We evaluated the energy resolution of the detector using the $2615 \mathrm{keV}^{208} \mathrm{Tl}$ line during calibration runs. The harmonic mean of the FWHM values obtained by fitting the CUORE0 peaks, weighted by physics exposure, is $4.9 \mathrm{keV}$ (with a corresponding RMS of $2.9 \mathrm{keV}$ ). This result improves the CUORICINO performance (5.8 keV, RMS of $2.1 \mathrm{keV}$ ) and demonstrates that the CUORE goal of $5 \mathrm{keV}$ FWHM in the region of interest has been achieved. The results of CUORE- 0 in terms of background reduction are also very important: the value measured in the CUORE-0 region of interest $(0.058 \pm 0.004$ counts $/ \mathrm{keV} / \mathrm{kg} / \mathrm{y})$, compared to the CUORICINO one $(0.153 \pm 0.006$ counts $/ \mathrm{keV} / \mathrm{kg} / \mathrm{y})$, shows that the techniques employed for the detector construction have been very effective. Compared with CUORE-0, CUORE will take advantage of a cryostat built with higher radio-purity materials and a more efficient anti-coincidence selection: the background budget extrapolated from CUORE-0 indicates that the goal of 0.01 counts $/ \mathrm{keV} / \mathrm{kg} / \mathrm{y}$ for CUORE is well within reach.

\section{3. $0 v \beta \beta$ searches with CUORE-0}

The CUORE- 0 energy spectrum in the region of interest is shown in Fig. 1 for the abovementioned exposure of $9.8 \mathrm{~kg}$ yr of ${ }^{130} \mathrm{Te}$. The peak closest to the $\mathrm{Q}$ value of the neutrinoless double beta decay of ${ }^{130} \mathrm{Te}$ is at $2506 \mathrm{keV}$; it is due to the single-crystal coincidence of the two $\gamma$ lines from the ${ }^{60} \mathrm{Co}$ decay. ${ }^{60} \mathrm{Co}$ is due to cosmic activation of copper frames and the internal shielding of the tower before transportation underground. We performed [8] a simultaneous fit of the unbinned data for each bolometer and each dataset using a 3-component function that contains: the hypothetical $0 v \beta \beta$ signal at $2527 \mathrm{keV}$, the peak from the ${ }^{60} \mathrm{Co} \gamma$ lines and a flat background, attributed to multi scatter Compton events from ${ }^{208} \mathrm{Tl}$ and surface $\alpha$ events. We found no evidence for $0 v \beta \beta$ decay of ${ }^{130} \mathrm{Te}$ and the best fit value for this decay rate is $\Gamma_{0 v}=0.01 \pm 0.12$ (stat.) \pm 0.01 (syst.) $\times 10^{24} \mathrm{yr}^{-1}$. Using a Bayesian approach, we set a $90 \%$ C.L. lower bound on the decay half-life of $2.7 \times 10^{24}$ yr. Combining this result with the $19.75 \mathrm{~kg}$ yr of ${ }^{130} \mathrm{Te}$ data from the CUORICINO experiment we set a global lower limit of $4.0 \times 10^{24} \mathrm{yr}$ at $90 \%$ C.L. (Bayesian): this is the most stringent limit up to date on the half life of $0 v \beta \beta$ decay of ${ }^{130} \mathrm{Te}$. The corresponding upper bound on the effective 
neutrino Majorana mass is in the range $270-650 \mathrm{meV}$, considering the most recent nuclear matrix element calculations in the context of $0 v \beta \beta$ decay mediated by light Majorana neutrino exchange and assuming $g_{A}=1.27$ for the axial coupling constant [8].

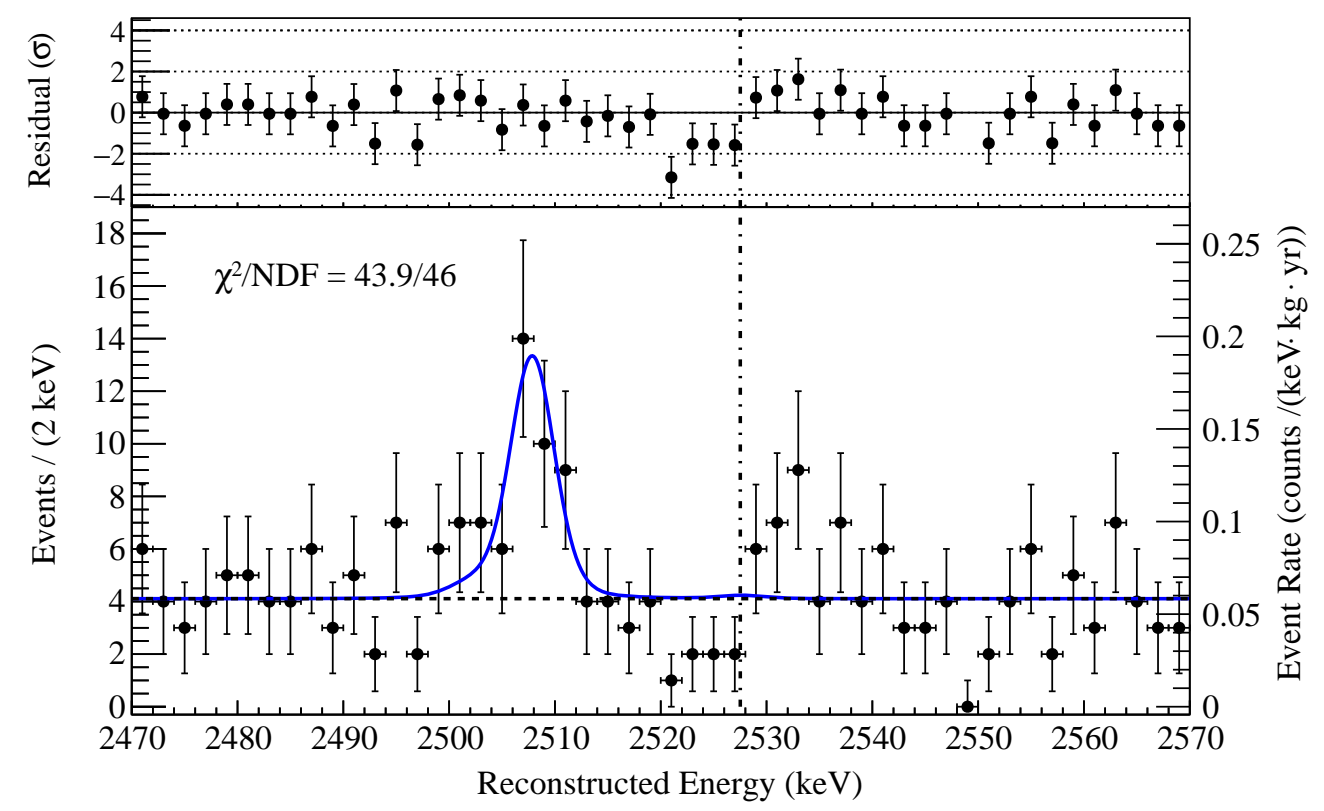

Figure 1: Bottom plot: the best-fit function (solid blue line) superimposed on the energy spectrum: the peak at $2507 \mathrm{keV}$ is attributed to ${ }^{60} \mathrm{Co}$; the dotted black line shows the continuum background component of the fit. The vertical dot-dashed black line indicates the expected position of $Q_{\beta \beta}$. Top plot: the normalized residuals of the best-fit model and the binned data points.

\section{CUORE}

The CUORE setup is an array of $988 \mathrm{TeO}_{2}$ crystals for a total mass of about $230 \mathrm{~kg}$ of ${ }^{130} \mathrm{Te}$. The crystals will be arranged in 19 towers, each with 13 floors ( 4 detectors per floor). The CUORE towers are identical to the CUORE- 0 tower described above. The whole array will be cooled down to $10 \mathrm{mK}$ in a cryogenic apparatus consisting of a custom made cryostat with six nested copper shields, a cryogen free cooling system based on five pulse tubes, a powerful dilution refrigerator, and a fast cooling system for pre-cooling. In order to shield the crystals against external radioactivity, the CUORE cryostat is surrounded by an outer lead shield and a neutron shield. In addition, an inner lead shield is positioned between the third and fourth copper shields and cooled to a temperature of $4 \mathrm{~K}$; it is made from ancient Roman lead with a very low contamination of ${ }^{210} \mathrm{~Pb}$. The construction of the experiment is now in its final stage. All towers have been assembled and are ready for the installation inside the cryostat. The external lead and neutron shields are in place. The cryostat has been mounted and tested at base temperature. In summer 2014 it reached the record temperature of $5.9 \mathrm{mK}$. Tests at full thermal load - i.e. including the inner lead shields - are ongoing and the start up of operations is expected in early 2016.

The expected CUORE sensitivity at $90 \%$ C.L. in 5 years is $T_{1 / 2}^{0 v}>9.5 \times 10^{25} \mathrm{y}$. It corresponds to a limit on the Majorana neutrino mass in the range $50-130 \mathrm{meV}$. 


\section{Conclusions}

2015 has been a very special year for CUORE. CUORE-0 achieved its energy resolution and background level goals, surpassing the CUORICINO sensitivity in half the time and showing that the CUORE design sensitivity is well within reach. CUORE-0 did not find evidence of ${ }^{130} \mathrm{Te}$ $0 v \beta \beta$ decay and, combined with the CUORICINO data, set the best limit to date on the half life of this decay. The CUORE experiment is in the final stage of construction at LNGS. All CUORE subsystems have been delivered and we plan to start operations in early 2016.

\section{References}

[1] B. Pontecorvo, Sov. Phys. JETP 26 (1968) 984

[2] A. Giuliani, A. Poves, Advances in High Energy Physics 2012, 857016, (2012).

[3] O. Cremonesi, M. Pavan, Advances in High Energy Physics, 2014, 951432, (2014).

[4] C. Arnaboldi et al., [CUORE Collaboration], Nucl. Instrum. Meth. A518 (2004) 775.

[5] R. Ardito et al., [CUORE Collaboration], hep-ex/0501010.

[6] E. Fiorini and T. O. Niinikoski, Nucl. Instrum. Meth. A224 (1984) 83.

[7] E. Andreotti et al., Astropart. Phys. 34 (2011) 822 .

[8] K. Alfonso et al. [CUORE Collaboration], Phys. Rev. Lett. 115 (2015) 102502 